\title{
Generalidades y aplicaciones de la instrumentación de pavimentos en condiciones de campo en Costa Rica
}

\section{Generalities and applications of pavement instrumentation with field conditions in Costa Rica}

\author{
Sheng Qi Wang Qiu \\ Unidad de Materiales y Pavimentos, LanammeUCR \\ Universidad de Costa Rica. San José, Costa Rica \\ sheng.wang@ucr.ac.cr
}

Fecha de recepción: 10 de junio de 2018 / Fecha de aprobación: 21 de septiembre de 2018

\section{RESUMEN}

La instrumentación de pavimentos en condiciones de campo es una de las metodologías que se encuentra aplicando el LanammeUCR para cumplir con el objetivo de estudiar y analizar los pavimentos de Costa Rica, con el propósito de mejorar su desempeño a mediano y largo plazo. La instrumentación se utiliza principalmente para determinar el comportamiento que presenta un pavimento a partir del efecto de cargas externas inducidas o fenómenos naturales y esto permite extender su aplicación a diferentes usos: monitoreo estructural, generación de modelos matemáticos, desarrollo de pruebas para validación de diseños, validación de otros métodos de estudio, entre otros. Por lo general dentro del área de ingeniería de pavimentos, el comportamiento que se adquiere corresponde a la respuesta (esfuerzos y deformaciones) del pavimento, que es registrada por la instrumentación utilizada en estos, en donde esta información podrá ser utilizada para las aplicaciones mencionadas anteriormente. En este estudio se presentan los procedimientos y los resultados preliminares que ha realizado y obtenido el LanammeUCR con respecto a la instrumentación de pavimentos en campo para Costa Rica.

PALABRAS CLAVE: pavimentos, instrumentación, esfuerzos, deformaciones, condiciones de campo

\section{ABSTRACT}

Pavement instrumentation underfield conditions is one of the methodologies that LanammeUCR is currently applying to meet its objectives of studying and analyzing Costa Rica's pavements with the purpose of improving their performance in the medium and long term. Pavement instrumentation is mainly used to determine the behavior a pavement shows under the effects of externally induced loads or natural phenomena, which allows a diverse range of uses: pavement structural monitoring, generation of mathematical models, development of tests for the validation of pavement designs, validation of other study methods, among others. Generally, in the field of pavement engineering, the acquired behavior corresponds to the response (stresses and strains) of the pavement, which is registered through the instrumentation used in these structures and the acquired information can be used in the applications mentioned before. This study shows the procedures and preliminary results that LanammeUCR has applied and acquired regarding the instrumentation of pavements under field conditions in Costa Rica.

KEYWORD: pavements, instrumentation, stresses, strains, field conditions 


\section{INTRODUCCIÓN}

Uno de los mayores problemas de infraestructura vial que presenta Costa Rica es el deteriorado estado en el que se encuentran los pavimentos en las vías del país. Una de las causas de esta problemática (además de prácticas indebidas durante el proceso constructivo, poco control durante las inspecciones y análisis deficientes de desempeño), es debido al uso de guías de diseño no apropiadas para las condiciones que se presentan en Costa Rica, en donde se tienen limitaciones en cuanto a las características de desempeño y deterioro de los materiales que se utilizan, diferentes condiciones climáticas y de tránsito.

Uno de los esfuerzos para solucionar esta problemática, proviene de parte del Laboratorio Nacional de Materiales y Modelos Estructurales de la Universidad de Costa Rica (LanammeUCR), el cual tiene como objetivo a largo plazo el desarrollo de una Guía de Diseño Estructural Mecanística Empírica de Pavimentos aplicable y actualizada para Costa Rica, en donde proyectos y estudios como el presentado en este artículo, forman parte de los esfuerzos de la institución para el desarrollo de esta. Cabe destacar que los resultados obtenidos hasta el momento para proyectos como el presentado en este artículo, son actualmente de carácter preliminar, por lo que el aporte de estos para el desarrollo de la guía se dará en el futuro.
El uso de una guía de diseño de este tipo no se centra sólo en calcular los espesores requeridos para una propuesta de un pavimento, si no que utiliza información del sitio y materiales donde se construirá y conformará este: condiciones de tránsito, de clima, características de los materiales que se utilizarán para el pavimento y sus posibles espesores. Esta información es utilizada para estudiar el comportamiento de su respuesta (esfuerzos, deformaciones y deflexiones) y el desempeño que tendrá el pavimento bajo esas condiciones. (Loría, 2013)

Seguidamente, se determina si el pavimento propuesto cumple con los criterios de desempeño (como fatiga, deformación permanente y daño por humedad) deseados para la vida útil esperada y las condiciones del sitio en donde se desea colocar. En caso de que no se acepte el diseño, se reinicia el proceso de diseño, cambiando los espesores propuestos o las propiedades de los materiales utilizados, realizando el proceso hasta que se acepte el desempeño obtenido. (Loría, 2013)

Como puede notarse, el uso de una guía de diseño de este tipo brindará pavimentos que se ajusten a las características y propiedades de los materiales de cada proyecto en específico. Para el caso de un país como Costa Rica, en donde se tiene gran variabilidad climática y distintos tipos de materiales, es necesario realizar diseños de este tipo para minimizar los factores que afectan el desempeño de los pavimentos y reducir la problemática actual en esta área. La Figura 1 muestra de forma resumida el proceso de diseño con una guía Mecanística - Empírica.

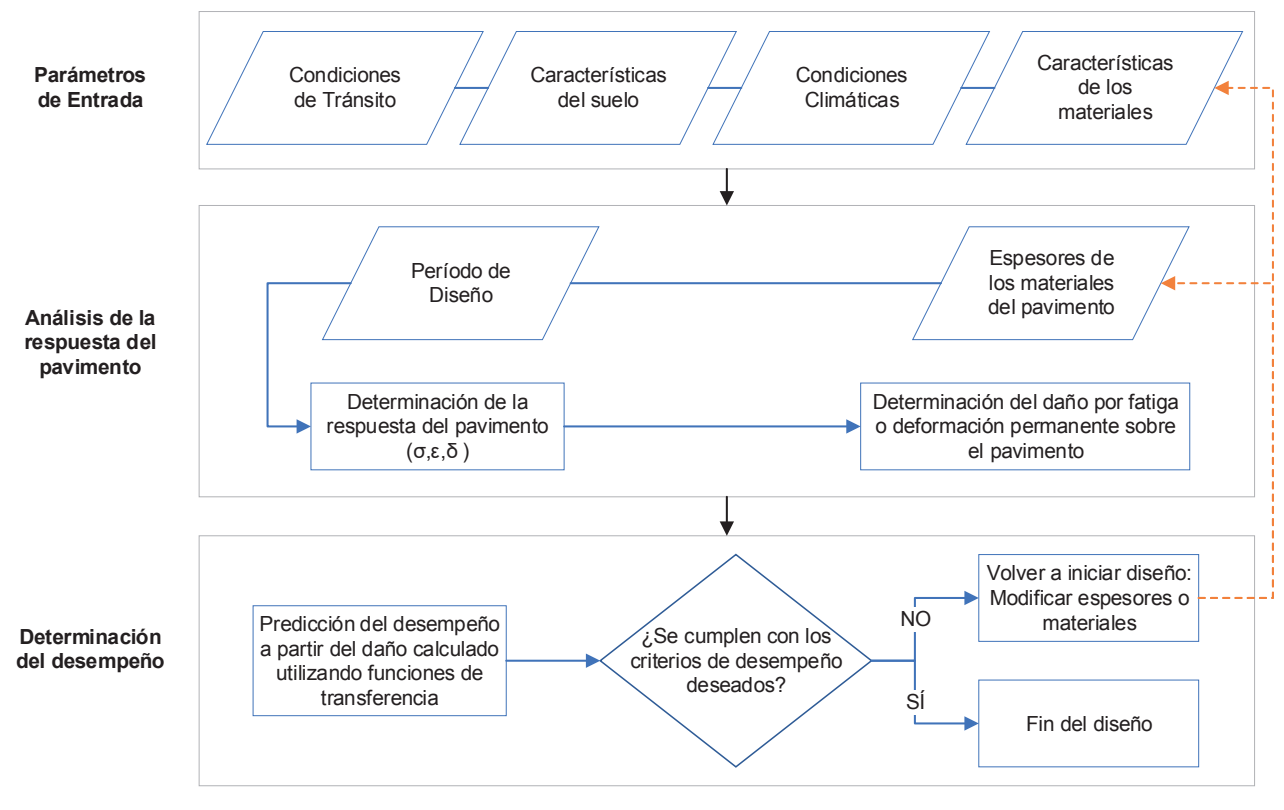

Figura 1. Procedimiento de diseño Mecanístico - Empírico

(Loría, 2013) 
Para que la Guía de Diseño Estructural Mecanística - Empírica de Pavimentos se adapte a las condiciones del país, es necesario generar indicadores, modelos matemáticos y ecuaciones propios que determinen de forma precisa los daños al pavimento $\mathrm{y}$ funciones de transferencia adecuadas para medir y predecir el desempeño del pavimento a largo plazo. Esto se logra de forma empírica, monitoreando los cambios que sufre un pavimento a lo largo de su vida útil. En la actualidad, para realizar este tipo de monitoreos, puede darse la instrumentación de tramos de campo que entrarán en servicio y estudiarlos a lo largo de su vida útil o mediante el uso de Ensayos Acelerados en Pavimentos (APT por sus siglas en inglés), los cuales permiten simular las fallas de toda la vida útil de un pavimento en tan solo unos meses (Aguiar, et al., 2012)

El LanammeUCR eligió inicialmente la línea de ensayos APT (después de un largo proceso de análisis y comparación de opciones), y adqduirió un Simulador de Vehículos Pesados (HVS por sus siglas en inglés) para realizar los estudios correspondientes al daño que sufren los pavimentos y las funciones de transferencia en estos. Aun así, aunque los ensayos APT sean a escala natural y busquen simular la realidad, siempre existirán condiciones controladas de laboratorio, además, son ensayos muy agresivos sobre el pavimento debido a su naturaleza de ser un ensayo acelerado. Por lo que para los modelos o ecuaciones que se logren determinar, podría darse una calibración u optimización adicional al compararse con información similar obtenida de campo.

Debido a lo anterior, el LanammeUCR decidió complementar los estudios actuales con el monitoreo e instrumentación de pavimentos en tramos que se encuentran actualmente en servicio, con el objetivo de realizar un seguimiento en el tiempo de estos y complementar la información nueva con de los ensayos APT. Debido a esto, el presente trabajo muestra las generalidades, procedimientos y aplicaciones que el LanammeUCR ha desarrollado y continuará desarrollando mediante la instrumentación de pavimentos en campo.

\section{INSTRUMENTACIÓN DE PAVIMENTOS EN CAMPO}

\section{Antecedentes}

La instrumentación de pavimentos en servicio en condiciones de campo es una de las formas que pueden ser utilizadas para el estudio de pavimentos por medio del análisis mecanístico - empírico, por lo que su aplicación no es nueva. A nivel internacional existen proyectos como el Long Term Pavement Performance (LTPP) en el cual instrumentaron proyectos con pavimentos rígidos y pavimentos flexibles que entrarían en servicio. Los monitoreos de estos tramos lo realizaron entre los años 1996 y 1999 y la información adquirida fue utilizada para profundizar los conocimientos en el campo del comportamiento de pavimentos ante las cargas externas que transitan sobre estos. (FHWA, 1999)

Otro proyecto a nivel internacional destacable es el proyecto MnROAD, que se encuentra actualmente activo y es desarrollado por el Departamento de Transporte de Minnesota, en donde realizaron la instrumentación de carreteras interestatales mientras se encontraban en su proceso de construcción. El monitoreo de estos tramos se realizó para observar el comportamiento de los pavimentos debido a condiciones ambientales en las que se encuentra y por diferentes volúmenes de tránsito. (MnROAD, 2015)

Una de las razones por las que existen proyectos de este tipo a nivel internacional y forman parte del interés del LanammeUCR es el potencial que presentan para mejorar el estudio y entendimiento del comportamiento de los pavimentos según las condiciones en las que se encuentren. En donde con la instrumentación de estos, puede estudiarse los efectos de cada variable (carga, temperatura, propiedades de los materiales $\mathrm{u}$ otros) que afectan las respuestas del pavimento en condiciones de campo.

\section{Instrumentación de proyectos nacionales}

Para avanzar en esta línea de investigación, el LanammeUCR ha buscado proyectos que se encuentren en la etapa constructiva y que permitan colocar la instrumentación de interés en las diferentes capas de los pavimentos. Al mes de Julio en el año 2018, se ha logrado realizar la instrumentación de un tramo de pavimento rígido en el Aeropuerto Internacional Juan Santamaría, un tramo en la ruta Cañas - Liberia (Ruta Nacional 1) y otro tramo en la ruta La Abundancia - Florencia (Ruta Nacional 35), correspondiente a un pavimento flexible.

Por el momento, el tramo con la mayor cantidad de información registrada y adquirida corresponde al del Aeropuerto Internacional Juan Santamaría (AIJS), por lo cual los detalles mostrados en el presente trabajo se enfocarán en este proyecto de instrumentación en particular. En este proyecto se instrumentó una losa de concreto, correspondiente a un pavimento rígido, en donde se utilizaron celdas de presión, galgas extensiométricas y termocuplas para su instrumentación. El uso que se le brinda a cada sensor se detalla a continuación: 


\section{- Celdas de Presión}

Las celdas de presión son utilizadas para medir los esfuerzos verticales que llegan hasta el nivel en donde se encuentra instalada la celda. Este tipo de mediciones se realiza para determinar en qué medida un pavimento distribuye y reduce los esfuerzos respecto a la profundidad de interés, por lo que su uso se da cuando existe una carga externa que transita sobre el pavimento.

En el caso de pavimentos rígidos, el uso de celdas de presión puede extenderse para analizar los efectos de alabeo de temperatura que se dan en estos pavimentos, ya que la misma losa de concreto causará un aumento o disminución del esfuerzo vertical en un punto en particular debajo de ella, que puede ser registrado si se poseen sensores a ese nivel.

Para casos con monitoreos y seguimientos en el tiempo del mismo pavimento, puede ser eventualmente posible, observar un incremento en los registros del esfuerzo vertical, lo cual indica cierto nivel de deterioro sobre la estructura y no logre reducir el esfuerzo vertical ante una carga como lo realizaba en su inicio (Leiva \& Camacho, 2015). Lo anterior corresponde a una de las aplicaciones que se realizan en la actualidad con la instrumentación de tramos de campo y ensayos APT. En la Figura 2 puede observarse una celda de presión modelo Geokon 3500-2, el cual tiene un rango de medición de 0 a 400 kPa.

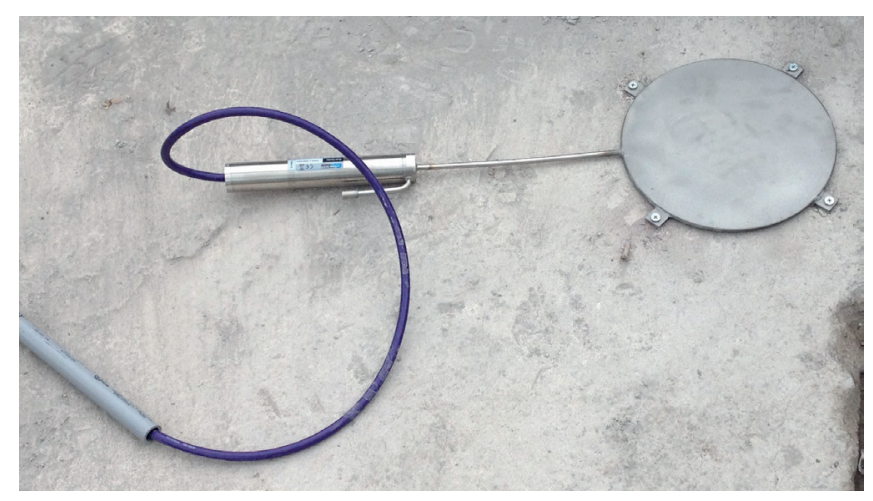

Figura 2. Celda de presión utilizada para la instrumentación de pavimentos (LanammeUCR, 2015)

\section{- Galgas Extensiométricas}

Las galgas extensiométricas son utilizadas para medir la deformación unitaria que experimenta un elemento estructural como un pavimento. Estos se pueden colocar con una orientación transversal o longitudinal, con el fin de conocer la deformación que se puede dar en esos sentidos. La deformación unitaria se desea obtener debido a que es utilizada para verificar los daños por fatiga que pueden darse en el pavimento. Por lo general tiene forma de "I" o " $\mathrm{H}$ " para lograr un anclaje adecuado con la estructura a medir. En la siguiente figura es posible observar este tipo de sensor para mediciones en concreto, ya que posee un soporte de acero en lugar de nylon (utilizado para mezclas asfálticas). Generalmente estos sensores permiten medir hasta los $1500 \mu \mathrm{s}$ (microstrain).

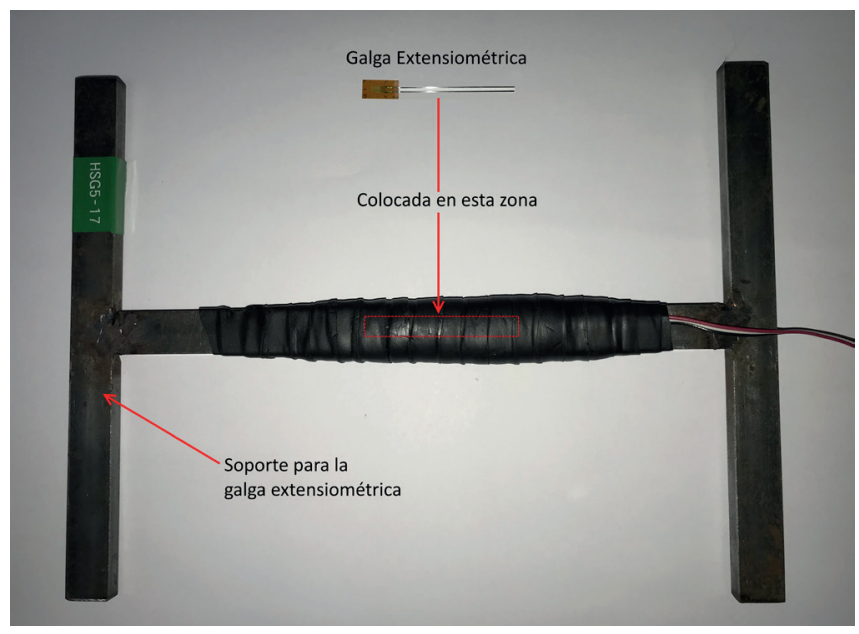

Figura 3. Galga extensiométrica

Otro de los usos en los cuales se utiliza la información de la deformación unitaria, es en la generación y validación de los modelos de daño y criterios de falla que son utilizados en una guía de diseño Mecanístico - Empírico (Leiva \& Camacho, 2014).Por ende, al igual que con las celdas de presión, en monitoreos periódicos de la vida útil de la estructura, puede esperarse un incremento en los registros de deformación debido al deterioro de la estructura. Debido a lo anterior, el uso de este tipo de sensores resulta primordial en una instrumentación de pavimentos, debido a los objetivos que tiene el LanammeUCR.

\section{- Termopares}

Los termopares son sensores utilizados para registrar la temperatura en donde se encuentran, lo cual puede ser aplicado para obtener la temperatura del ambiente, en la superficie del pavimento o a un nivel debajo de este. En pavimentos rígidos, conocer la temperatura a un nivel cercano en la superficie y a un nivel cercano al inferior es importante para realizar un estudio enfocado a los efectos de alabeo por temperatura que estos sufren. De esta manera, puede relacionarse la temperatura con la respuesta que obtienen los otros sensores utilizados (esfuerzo vertical y deformación). 
Para el caso de pavimentos flexibles, la temperatura es una de las variables que afecta el módulo de dinámico en la carpeta asfáltica, lo cual es uno de los indicadores del comportamiento que estos pueden tener (mayor o menor rigidez), lo cual afectará los esfuerzos y deformaciones registrados por los otros sensores. En la Figura 4 puede observarse un ejemplo de un termopar tipo K (compuesta por dos filamentos: uno de Nickel - Cromo y otra de Nickel - Aluminio) utilizada para la instrumentación.

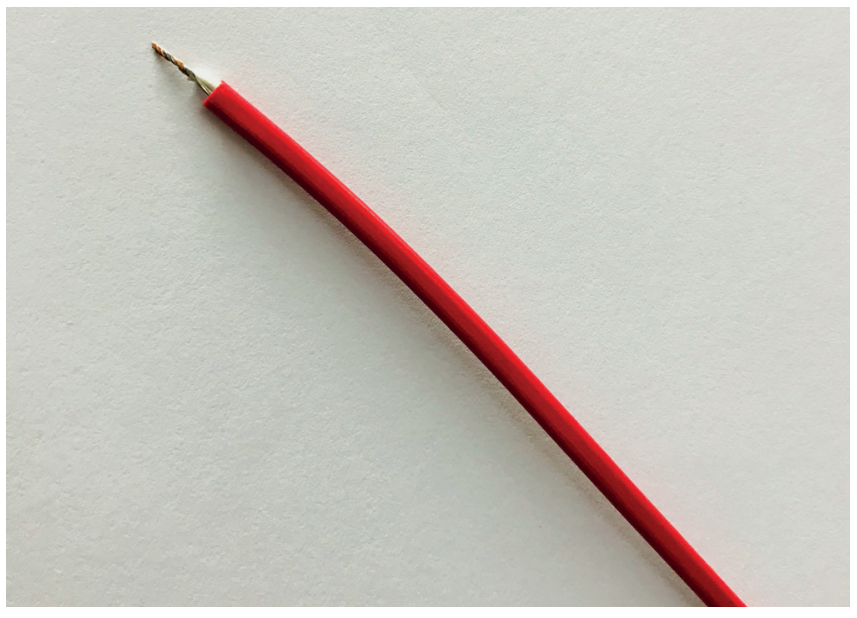

Figura 4. Termopar tipo K

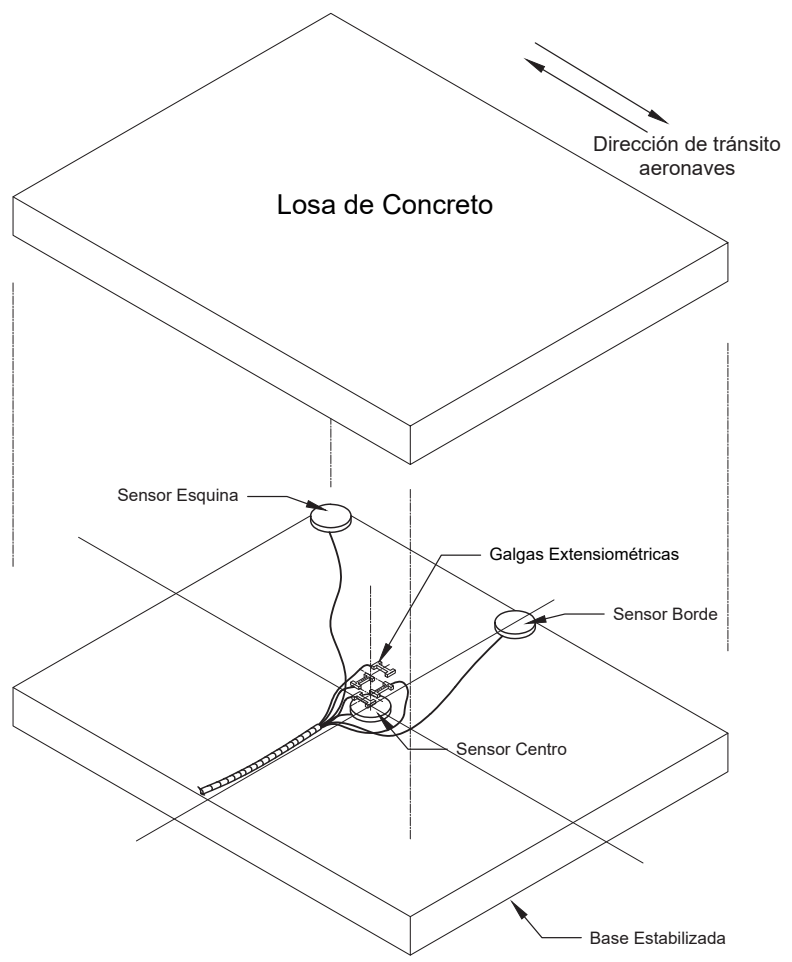

\section{Configuración de la instrumentación en el Aeropuerto Internacional Juan Santamaría}

Para el pavimento rígido del AIJS, una losa de concreto con dimensiones de 4.0 x $5.0 \mathrm{~m}$ fue instrumentada con la siguiente cantidad de sensores: 3 celdas de presión (esquina, centro y borde), 4 galgas extensiométricas y 3 termopares. El posicionamiento de estos sensores dentro de la losa de concreto puede observarse en las siguientes figuras:

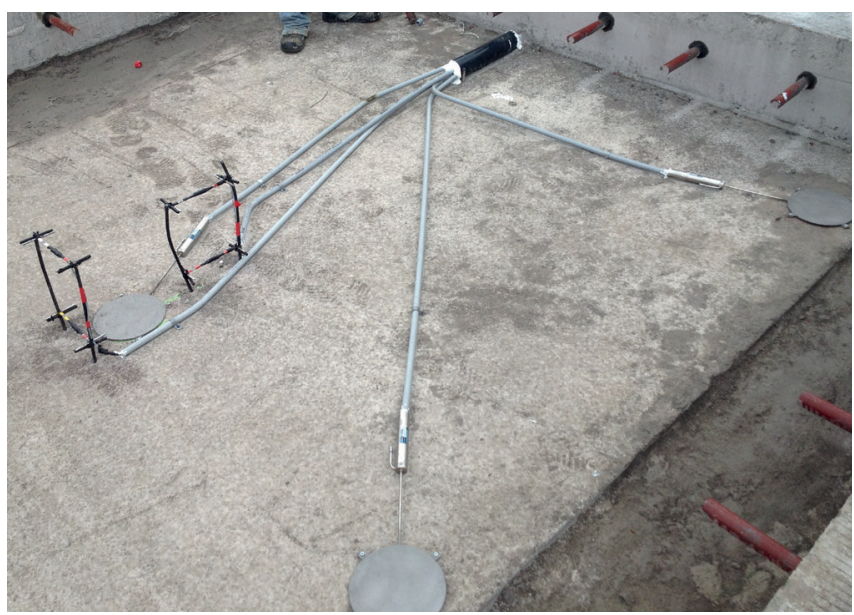

Figura 5. Instrumentación del pavimento rígido del AlJS (LanammeUCR, 2015)

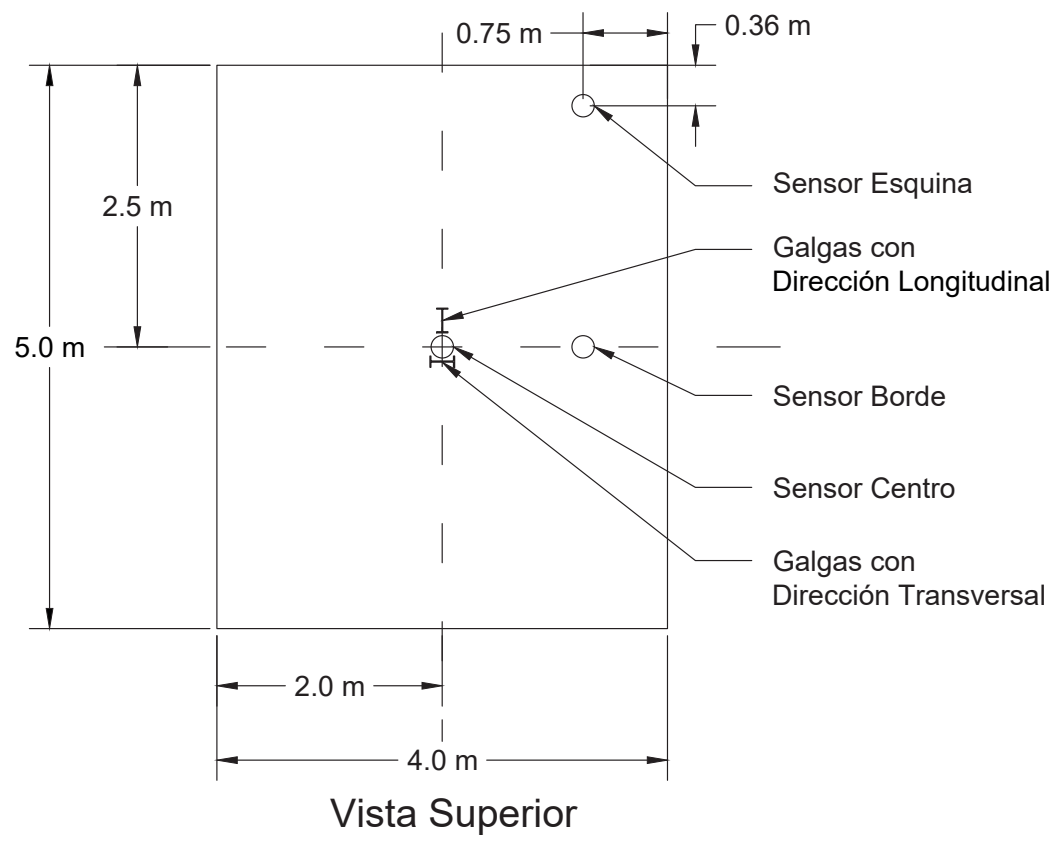

Figura 6. Vista Isométrica y Superior de la losa de concreto instrumentada y los sensores utilizados

(LanammeUCR, 2015) 
Con la colocación de los sensores de la forma observada, es posible obtener el registro de los esfuerzos verticales para las posiciones de Esquina, Centro y Borde de la losa cuando se dé el tránsito de un vehículo (respuesta debido a una carga externa). Asimismo, también se posibilita la adquisición de la respuesta del pavimento en estas posiciones debido a los efectos de alabeo por temperatura.

Las galgas extensiométricas fueron instaladas en ambas direcciones axiales (longitudinal y transversal) en la zona central de la losa. De esta forma es posible obtener la deformación unitaria que se da en ambas direcciones debido a cargas externas o a los efectos de alabeo por temperatura. Además, estas galgas extensiométricas se colocaron en la posición cercana al borde superior y al borde inferior de la losa de concreto (como se observa en la Figura 7), para obtener las deformaciones que suceden a estos niveles por los esfuerzos de compresión y tensión que se dan.

Los termopares se encuentran al mismo nivel de las galgas, lo cual permite obtener la temperatura de estas zonas de la losa y determinar el diferencial térmico que existe, causante de los efectos de alabeo comunes en los pavimentos rígidos. El tercer termopar no se encuentra dentro de la losa, ya que está siendo utilizada para obtener la temperatura del ambiente, sin embargo, se encontraba inhabilitada en el momento de las mediciones debido a problemas de conexión en el sensor.

\section{RESPUESTA DE LOS PAVIMENTOS INSTRUMENTADOS}

Con la instrumentación realizada, la respuesta de los pavimentos que puede ser adquirida corresponde a la del esfuerzo vertical y la deformación unitaria. En el caso del AIJS, se busca la respuesta generada por la aeronave A340-600, al ser una de las naves con una configuración de llantas más crítica (mayor carga sobre cada llanta en comparación a otras aeronaves) que arriban al aeropuerto con una carga máxima para su despegue de 365 ton (Airbus, 2017).

Para este proyecto, la adquisición de la respuesta de este pavimento se ha enfocado solo en el esfuerzo vertical, debido a las maniobras y forma de transitar realizado por la aeronave en estudio. La respuesta que se espera obtener corresponde al crecimiento y decrecimiento de una curva conocida como el pulso de carga que se genera a partir de una carga dinámica externa. Esto se ejemplifica en la Figura 8, asumiendo el paso de una llanta por el centro de la losa, en donde la respuesta es adquirida por una celda de presión.

La forma de la curva el pulso de carga será similar para las galgas extensiométricas, ya que su forma se detección es asemeja a las de las celdas de presión, pero la información que adquieren es completamente distinta (deformaciones en lugar de esfuerzos). En el caso de los efectos de alabeo por temperatura, se espera que para un período de 24 horas se observen incrementos y decrementos de esfuerzos verticales registrados debido al tipo de alabeo que se genere por las temperaturas durante el día y la posición del sensor. Con un alabeo hacia abajo, las posiciones de Borde y Esquina tendrán un incremento en los esfuerzos, mientras que la posición de Centro tendrá un decremento. Con un alabeo hacia arriba, se obtiene el efecto inverso. Lo anterior puede visualizarse en la Figura 9.

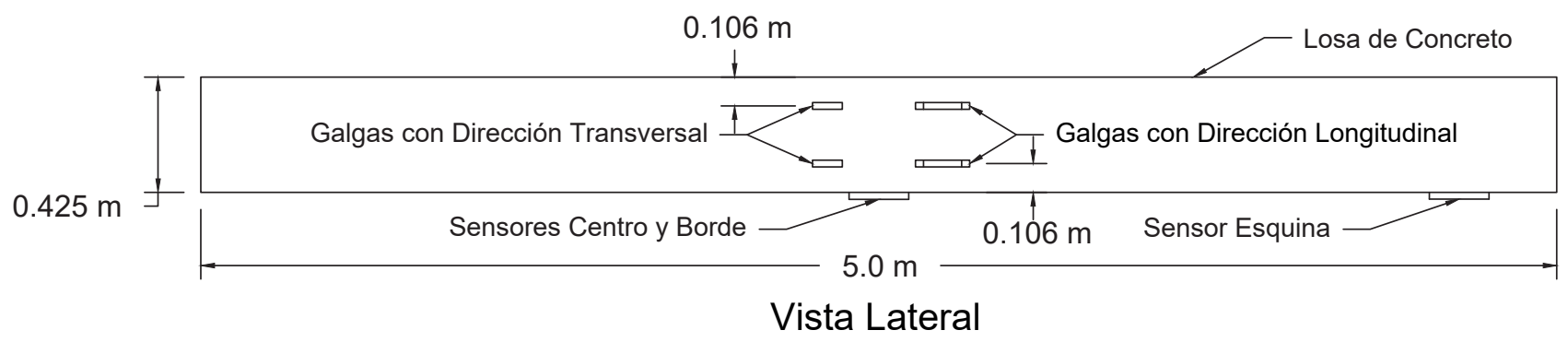

Figura 7. Vista Lateral de la losa de concreto instrumentada y los sensores utilizados (LanammeUCR, 2015) 

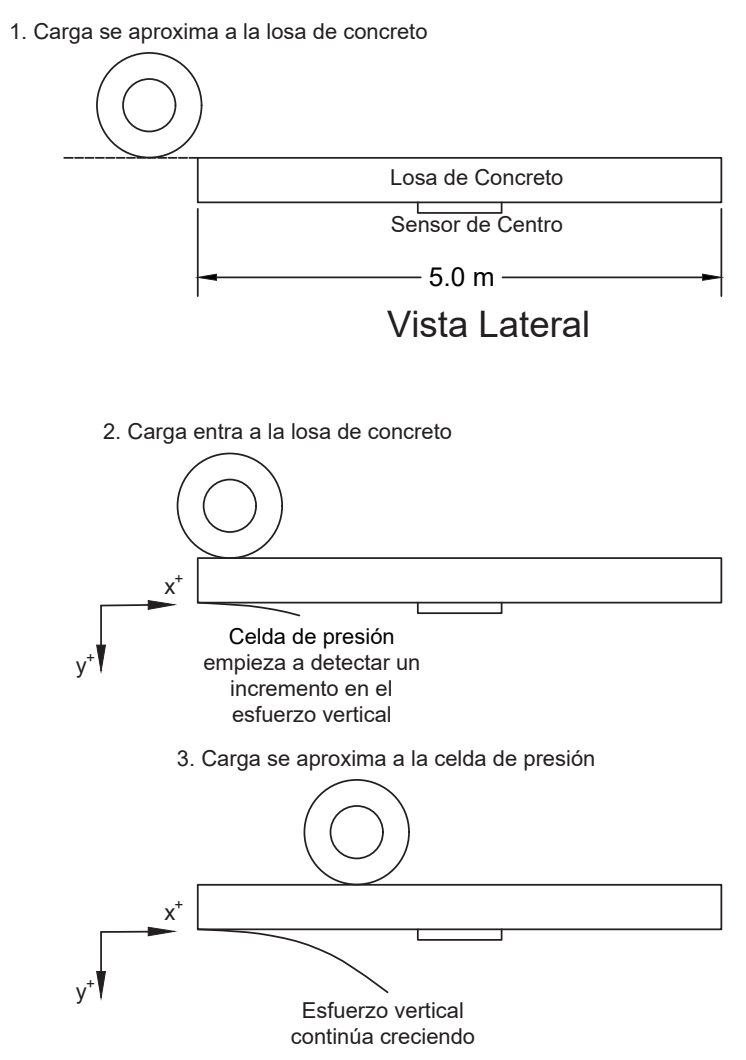
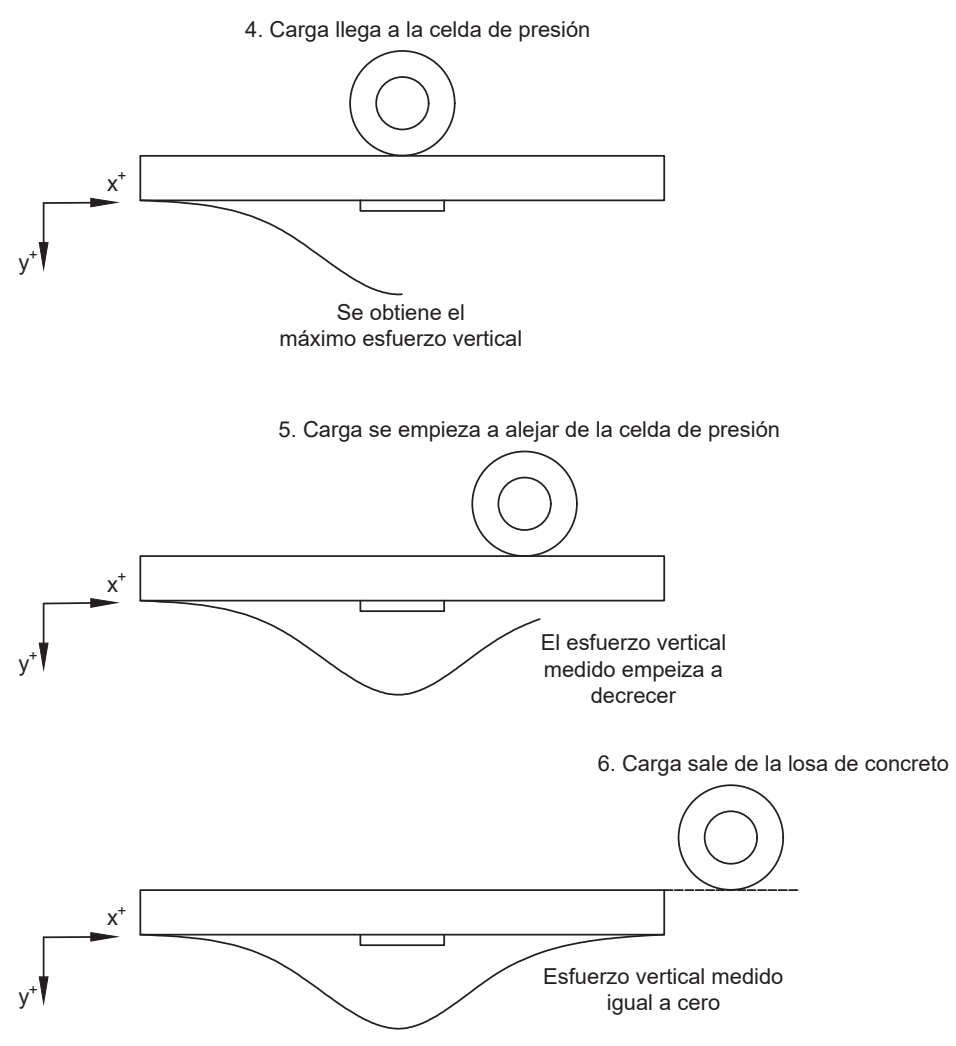

Figura 8. Forma de la respuesta generada por una carga externa
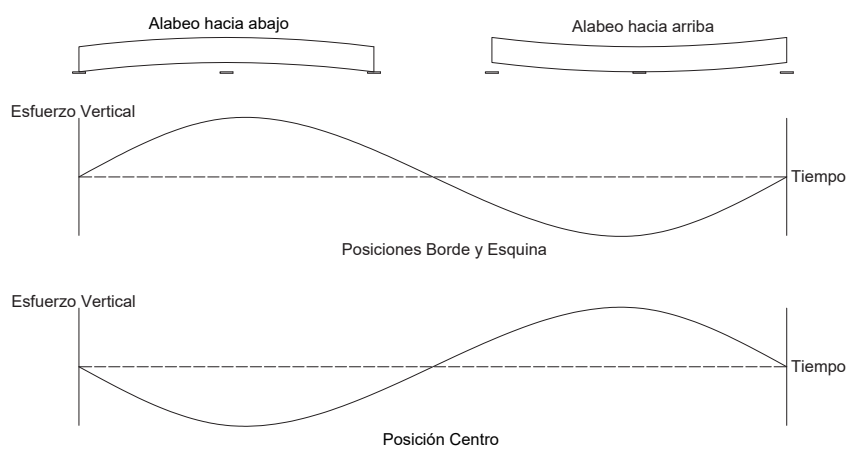

Conociendo las formas de las respuestas esperadas, se facilita la interpretación e identificación de la información generada en campo, ya que puede determinarse si los datos adquiridos son los correctos o no. Por ejemplo, para la respuesta generada por una carga externa, se ha obtenido información como la mostrada en la Figura 10.

Figura 9. Forma de la respuesta generada por efectos de alabeo 


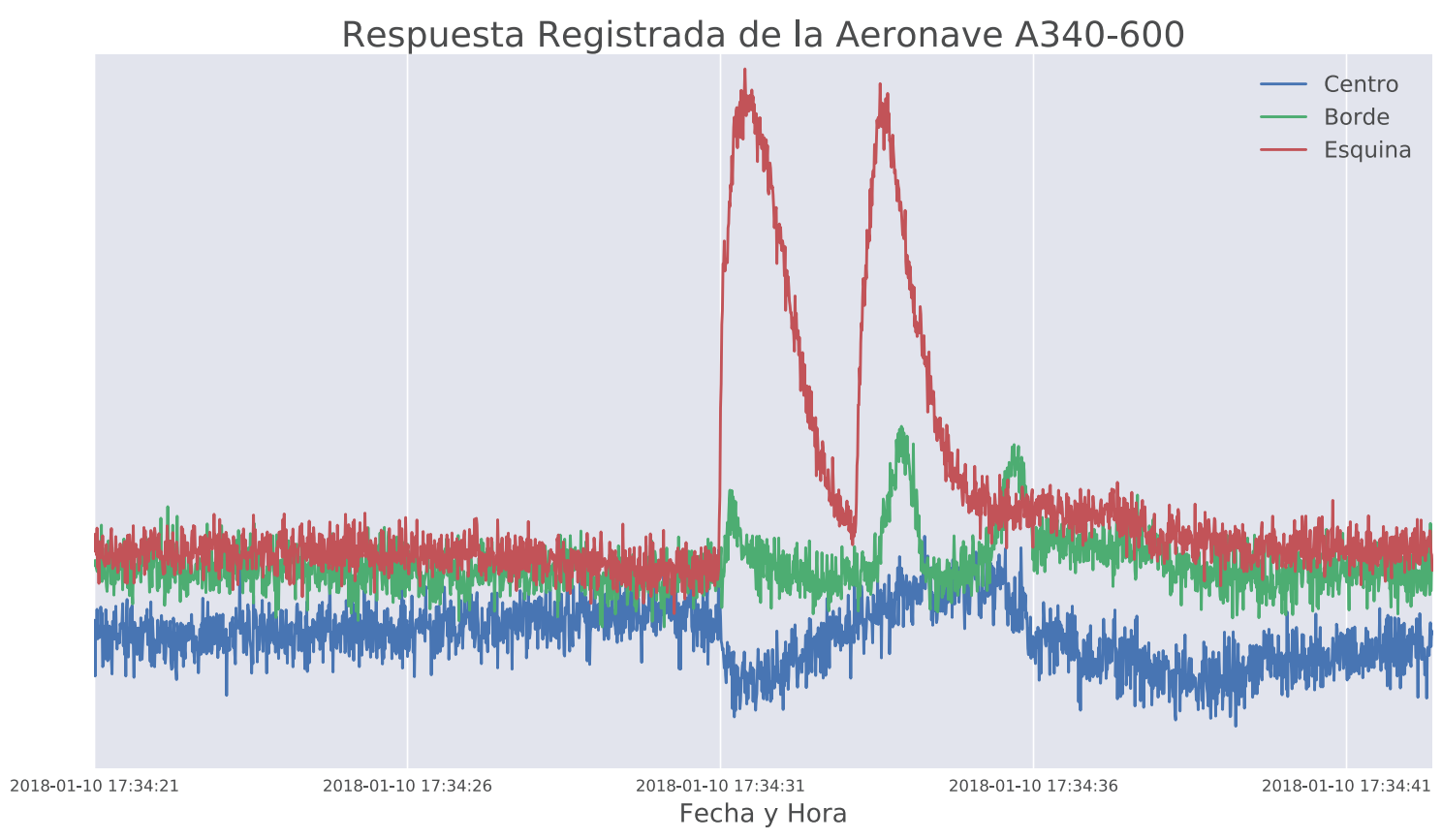

Figura 10. Respuesta del esfuerzo vertical obtenido para el tránsito de la aeronave A340-600

La celda de presión en la posición de Esquina es la que mostró un esfuerzo vertical mayor, esto debido a que las llantas de la aeronave A340-600 transitan principalmente sobre la zona en la que se encuentra este sensor. Otro aspecto por observar es que existen dos curvas consecutivas con la forma esperada de la respuesta, lo cual es debido a la configuración de las llantas (eje tándem) del vehículo en estudio que transita por la losa instrumentada esto se muestra en la Figura 11.

Con respecto a los efectos de temperatura, la respuesta generada (esfuerzos verticales) por los efectos de alabeo puede ser observada en la Figura 12, en donde puede notarse que se logra observar los incrementos y decrementos de esfuerzo vertical debido a los tipos de alabeo que la losa de concreto sufrió durante un período de 24 horas. Las temperaturas que provocan estos alabeos y cambios en el esfuerzo vertical se observan en la Figura 13, donde se tiene el registro de las 24 horas para las temperaturas de la zona superior e inferior de la losa.

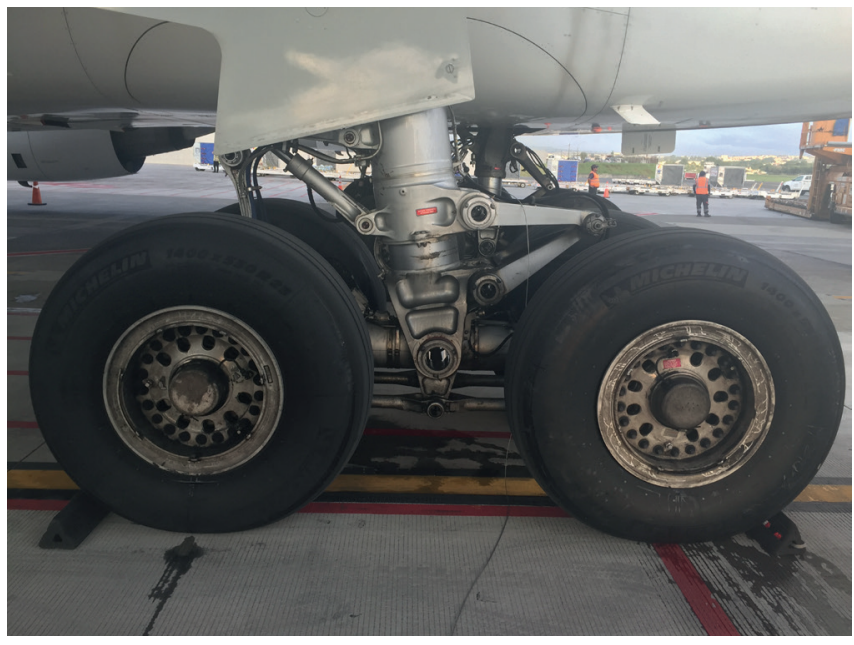

Figura 11. Configuración de las llantas de la aeronave en estudio A340-600 


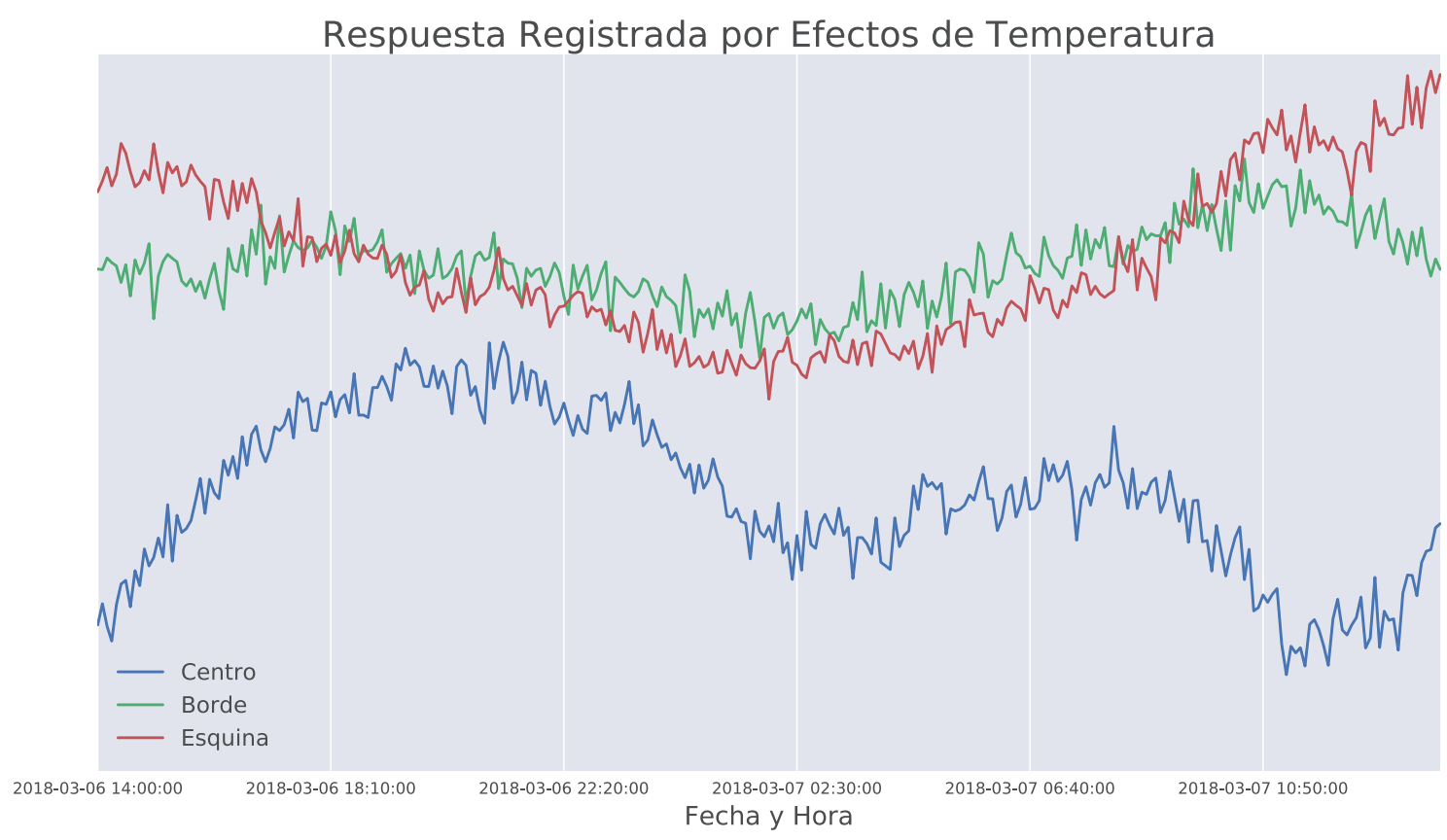

Figura 12. Respuesta del esfuerzo vertical generado por los efectos de alabeo en un período de 24 horas

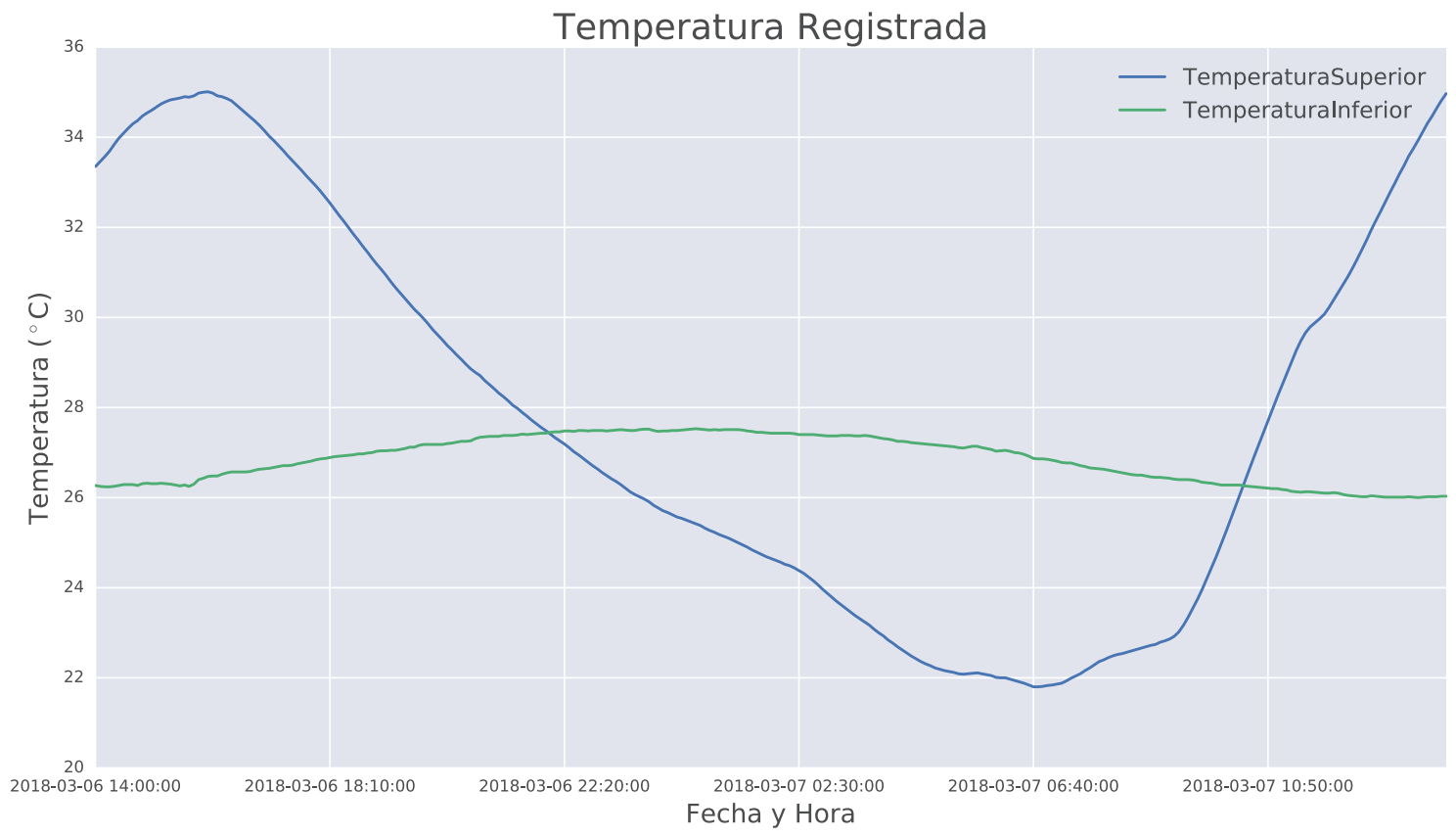

Figura 13. Temperaturas registradas en la parte superior e inferior de la losa de concreto a una profundidad de $10,6 \mathrm{~cm}$ de los extremos 


\section{APLICACIONES DE LA INSTRUMENTACIÓN DE PAVIMENTOS EN CAMPO}

Dentro de las aplicaciones para los cuales se desea utilizar la instrumentación realizada se destacan los monitoreos y seguimientos periódicos en el tiempo de las estructuras de pavimento. En donde pueden registrarse los cambios en la respuesta a través del tiempo y relacionarlo con los deterioros que se den en el pavimento. Esto brinda la posibilidad de generar los modelos matemáticos utilizados para predecir los daños y desempeños de los pavimentos que son utilizados en guías de diseño Mecanístico - Empíricos.

El uso de la instrumentación de tramos en servicio en conjunto con los ensayos de tipo APT posibilitan la optimización de los modelos matemáticos de predicción de daño y desempeño generados por estos últimos. Combinando estas dos metodologías, se logra el desarrollo de estos modelos matemáticos en el corto plazo y la optimización de estas a largo plazo, mediante lo observado en condiciones de campo. La profundización de los conocimientos en el campo de los pavimentos también se da de forma conjunta al observar los resultados que brindan la aplicación de estos métodos, al darse la posibilidad de experimentar con diferentes variables que tengan efectos sobre este tipo de estructuras.

Otro uso que puede brindársele a los resultados obtenidos es para la verificación y comparación de los métodos de cálculo que se utilizan actualmente para determinar las respuestas estructurales que se dan en los pavimentos (esfuerzos y deformaciones). Entre algunos de estos métodos se destacan la modelación por el Método de Elemento Finito para pavimentos rígidos y la Teoría de Multicapa Elástica en pavimentos flexibles, en donde la comparación de los resultados brindados por estos métodos con mediciones de campo permite conocer la magnitud de aproximación que dan con respecto a la realidad, con lo que se puede validar el uso de estos métodos de cálculo.

Adicionalmente, con la información generada a partir de la instrumentación de pavimentos, puede lograrse la calibración de valores como gradientes térmicos o coeficientes de expansión térmica (para el caso de pavimentos rígidos) que actualmente se asumen de lo indicado por la teoría. Con valores calibrados de este tipo, se logran optimizar estas variables de diseño, adecuándolas a las zonas o proyectos en las que se desempeñaría un pavimento.

\section{CONCLUSIONES}

Aunque la instrumentación de pavimentos en condiciones de campo no es un método de estudio de pavimentos nuevo, su uso en la actualidad posee gran validez y muchas aplicaciones que son del interés del LanammeUCR como parte de sus objetivos para el desarrollo de la Guía de Diseño Estructural Mecanística - Empírica de pavimentos para Costa Rica.

Se ha observado que dentro de los usos que posee la instrumentación de pavimentos en campo se destacan: monitoreos estructurales, generación de modelos matemáticos para la predicción del daño y desempeño que poseen, optimización de estos modelos, validación de otros métodos de estudio, desarrollo de experimentos para la validación de diseños de pavimentos y sus variables, entre otros.

Al presentarle la oportunidad de obtener resultados deseados a partir de las instrumentaciones que se realizan y generar una amplia cantidad de aplicaciones posibles, continúa siendo del interés del LanammeUCR seguir y expandir el uso de esta metodología para lograr sus objetivos con respecto al estudio y análisis de los comportamientos que presentan los pavimentos en Costa Rica.

\section{REFERENCIAS BIBLIOGRÁFICAS}

1. Aguiar, J. P., Corrales, J. P., Elizondo, F., \& Loría, L. G. (2012). PaveLab and heavy vehicle simulator implementation at the National Laboratory of Materials and Testing Models of the University of Costa Rica. LanammeUCR, San José.

2. Airbus. (2017). A340-600 Aircraft Characteristics/Airport and Maintenance Planning. Francia.

3. FHWA. (1999). Introduction to LTPP Data. Virginia: U.S Department of Transportation.

4. $\quad$ LanammeUCR. (2015). Base de Datos Sobre Instrumentación de Pavimentos. San José.

5. Leiva, F., \& Camacho, E. (2014). LM-PI-UMP-028-R1: Instrumentación y resultados del primer ensayo acelerado a escala natural en Costa Rica. LanammeUCR, San José.

6. Leiva, F., \& Camacho, E. (2015). LM-PI-UMP-042-R1: Evaluación PAVELAB Tramos 002AC4. LanammeUCR, San José.

7. Loría, L. G. (2013). Desarrollo de la Guía de Diseño de Pavimentos de Costa Rica. LanammeUCR, San José.

8. MnROAD. (2015). Instrumentation and Data Collection at MnROAD. Obtenido de Minnesota Department of Transportation: http://www.dot.state.mn.us/ mnroad/calendar/STA2015Presentations/3\%20-\%20Instrumentation\%20and\%20Data\%20Gathering\%20(Palek).pdf 